\title{
SYSTEMS ANALYSIS OF THE HEMODYNAMICS IN TETRALOGY OF FALLOT: BASED ON FORTRAN IV COMPUTER SIMULATION*
}

\author{
A. Thomas Look, JR. $\dagger$ and Thomas R. Bates, JR. $\$$ \\ University of Michigan, Ann Arbor, Michigan 48104, U.S.A.
}

\begin{abstract}
A hemodynamic model has been developed for the patient with tetralogy of Fallot based on electrical circuit analog and oxygen balance equations. A computer program has been written for the model in the Fortran IV computer language. With the computer model it has been possible to simulate the physiologic conditions during exercise, squatting and hypoxic episodes in tetralogy of Fallot. In addition, it has been possible to simulate the palliative effect of systemic-to-pulmonary shunt operations.

Partial factorial analysis has been employed in the computer program to enable investigation of the physiologic interactions in tetralogy of Fallot. Graphical representations of the computer output are presented which demonstrate precise cause and effect relationships in exercise, squatting and hypoxic episodes. An explanation is advanced for the lack of correlation between resting arterial oxygen saturation and the incidence of hypoxic episodes.
\end{abstract}

\section{INTRODUCTION}

In the congenital heart disease tetralogy of Fallot there are two primary morphologic lesions--pulmonary valvular stenosis and a ventricular septal defect. Kirklin and Karp (1970) point out in their monograph, Tetralogy of Fallot, that it is worthwhile to limit the definition to those cases with a large ventricular septal defect approximating the size of the aortic valvular orifice' and pulmonary stenosis severe enough to cause right to left shunting. Within this definition there is classically a high anterior ventricular septal defect and pulmonary stenosis which is wholly or in part infundibular, due to a distinctive abnormal embryologic development of the truncus septum. Variants of the same embryologic picture which do not meet the criterion of right to left shunting can be more properly considered on a hemodynamic basis with uncomplicated ventricular septal defect anomalies (Kirklin and Karp. 1970).

Another key aspect of tetralogy of Fallot is the increase in aorto-pulmonary collateral circulation. This aorto-pulmonary shunt flow is described as both an enlargement of the normal precapillary anastamoses and the presence of small or mediumsized vessels joining the aorta to large branches of the pulmonary arteries. The collateral flow has been measured directly for patients with tetralogy of $\mathrm{Fal}$ lot during complete cardio-pulmonary bypass surgery. Moffit, Kirklin and Theye (1962) report an average aorto-pulmonary shunt flow of 22 per cent of the total cardiac output; Kirklin and Karp (1970) report a range of 5-40 per cent. Aorto-pulmonary shunt flow rates in the same range were also deter-

* Received 20 May 1974

† Present address: Department of Microbiology RM 6706 Medical Science II, Medical School.

$\ddagger$ Department of Mechanical Engineering. mined by indirect physiologic studies in earlier work by Bing et al. (1947).

The clinical syndrome of tetralogy of Fallot includes four well-recognized physiologic phenomena:

(1) the marked decrease in arterial oxygen saturation and the overall limitation of oxygen consumption which occur during exercise,

(2) the rapid increase in arterial oxygen saturation which occurs with squatting during recovery from exercise,

(3) the tendency for the development of characteristic hypoxic spells in approx. 40 per cent of patients, and

(4) the improvement that patients experience following palliative systemic-to-pulmonary shunt operations.

These phenomena are difficult to understand due to the complex interactions between normal adaptive physiologic responses and the abnormal central circulatory pattern in tetralogy of Fallot.

A computer model is developed which simulates the hemodynamic system in tetralogy of Fallot. Based on the principles of partial factorial analysis, the computer model is used to investigate the cause and effect relationships behind the physiologic phenomena in tetralogy of Fallot.

\section{METHODS}

Table 1 defines the notation which is used in the derivation of equations and in the presentation of data in the tables and figures which follow.

\section{Hemodynamic model of tetralogy of Fallot}

A schematic diagram which outlines the hemodynamic system in tetralogy of Fallot is presented 
Table 1. Computer model notation

Variables $\begin{aligned} P & =\text { pressure } \\ Q & =\text { blood flow rate } \\ R & =\text { resistance } \\ E & =\text { voltage } \\ I & =\text { current } \\ X & =\text { oxygen saturation (fractional) } \\ \mathrm{O}_{2} \mathrm{CON} & =\text { oxygen consumption } \\ \mathrm{H}_{b} \mathrm{CAP} & =\text { hemoglobin oxygen capacity } \\ A & =\text { aortic } \\ R H & =\text { right heart } \\ L H & =\text { left heart } \\ S Y & =\text { systematic } \\ S & =\text { aorto-pulmonary shunt } \\ D & =\text { ventricular septal defect } \\ P & =\text { pulmonary valve } \\ L & =\text { lung vessels } \\ I N F & =\text { pulmonary infundibulum } \\ M V R & =\text { mixed venous return } \\ K & =\text { mixing correlation constant }\end{aligned}$

in Fig. 1. This diagram shows the systemic venous return feeding the right atrium at point A. Blood leaving the right heart at point $\mathbf{B}$ is pumped in two directions. One portion of the flow, $Q_{D}$, is pumped through the ventricular septal defect and eventually into the aorta. The other portion of the blood flow, $Q_{P}$, is pumped through the stenosed pulmonary valve to point $C$, where it is joined by the aorto-pulmonary collateral shunt flow, $Q_{s}$. Together these two flows, $Q_{P}$ and $Q_{S}$, comprise the total flow through the lungs, $Q_{L}$, into the left atrium. This flow is now pumped into the aorta where it joins $Q_{D}$ at point $\mathrm{D}$ to form the total aortic blood flow, $Q_{A}$. The aortic flow now subdivides at point $E$ into the systemic flow, $Q_{S y}$, which later becomes the venous return at point $A$, and the collateral aortopulmonary shunt flow, $Q_{\text {s. }}$.

The two sides of the heart (shown separately for ease of schematic illustration) are represented hemodynamically as pumps, whereas blood-carrying vessels are represented as resistances to flow. For convenience, all of the blood vessels in the lungs have been lumped together as a single resistance, $R_{\mathrm{L}}$. Similarly, the resistance of the collateral shunt circulation is $R_{S}$; the resistance of the pulmonary artery and stenosed pulmonary valve is $R_{P}$; the resistance of the ventricular septal defect is $R_{D}$ and the systemic resistance is $R_{s Y}$. The aortic resistance is negligible because of the large diameter of the aorta, so it was not included in the model.

The basic law of blood flow relates the pressure drop, $\Delta P$, across an element to the flow resistance, $R$, and the blood flow rate, $Q$, as follows:

$$
\frac{\Delta P}{R}=Q \text {. }
$$

There are several assumptions (in the form of specific criteria to be met) inherent in the basic law. These

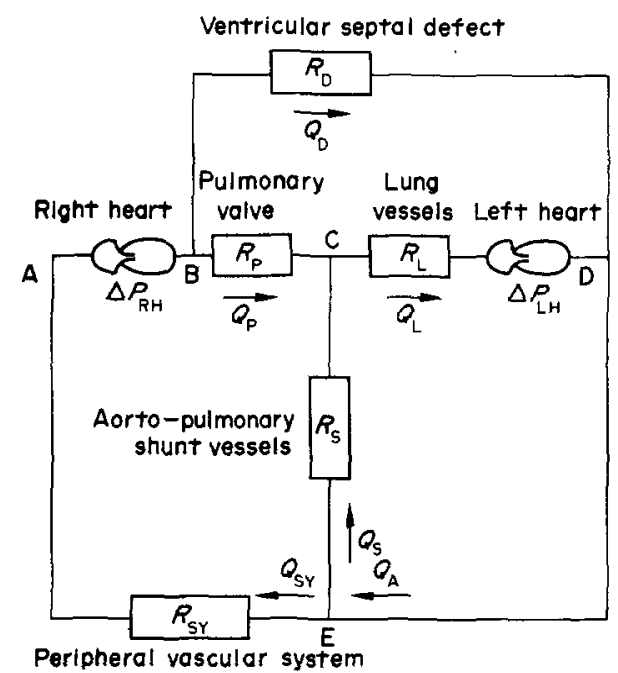

Fig. 1. Hemodynamic schematic diagram of tetralogy of Fallot.

assumptions, as they affect this model, are as follows:

(1) The flow is assumed to be steady so transient responses are neglected. The pumping action of the heart is assumed to be a quasi-equilibrium process with pressures and flow rates averaged over the period of systolic ejection only.

(2) The resistances to flow are assumed to be constant, so the values of the resistance are functions of geometry only. Therefore, the values do not change as the flow rates change.

(3) The vessel walls are assumed to be rigid, so all the elements are purely dissipative in nature. Consequently, the capacitive nature of the distensible venous vessels is overlooked as it leads only to transient effects which do not affect the equilibrium relationships of the basic law.

\section{Electrical circuit analog model}

According to well established principles of modeling theory, the hemodynamic flow model shown in Fig. 1 can be resolved into the electrical circuit ana$\log$ shown in Fig. 2 (Dorf, 1967). In this analog model the electrical resistors, $R_{D}, R_{L}, R_{P}, R_{S}$ and $R_{S Y}$, correspond to the resistances to fluid flow through the ventricular septal defect, the lungs, the stenosed pulmonary valve, the collateral aorto-pulmonary shunts, and the peripheral vascular system, respectively. The batteries shown in Fig. 2 correspond to the pumps of the right and left heart. The voltage differences in the electrical model, $E_{R}$ and $E_{L}$, are analogous to the pressure differences, $\Delta P_{R}$ and $\Delta P_{L}$, in the flow model. In the same manner the electrical current, $I$, corresponds to the hemodynamic flow, $Q$.

The solution equations of linear electric circuits are classically written in terms of independently defined loop currents (Scott, 1965). The electrical circuit model of tetralogy of Fallot contains three such independent loop currents, illustrated by $I_{1}, I_{2}$ and $I_{3}$, in Fig. 2 . The equations generated by these circuit 


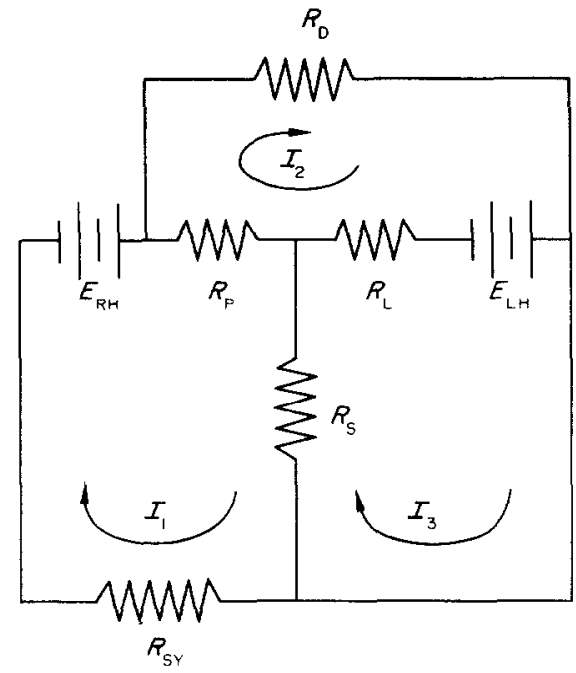

Fig. 2. Electrical circuit analog diagram of tetralogy of Fallot.

loops are expressed in terms of three-dimensional square matrices, and solved for $I_{1}, I_{2}$ and $I_{3}$. Solution of the matrices yields the equations shown in Table 2.

\section{Oxygen balance}

It is possible to derive independent equations for the oxygen balance in tetralogy of Fallot based on the hemodynamic flow model. One factor which complicates the oxygen mass balance is the mixing which occurs in the right and left ventricles across the ventricular septal defect (Kirklin and Karp, 1970). The net flow across the ventricular septal defect is from right to left due to the higher average pressure in the right ventricle during systolic ejection. There is a mixing flow from left to right, however, during the early portion of diastole. This means that saturated blood returning from the lungs mixes with unsaturated blood returning from the system prior to ventricular ejection. This mixing rules out a simple oxygen mass balance analysis.

In order to model this interventricular mixing a control volume is defined to include both ventricles as a unit, with two input and two output streams (see Fig. 3). Based on this model three independent oxygen balance equations shown in Table 3 are written. Equation (1) is the result of a simple oxygen balance on the input and output streams from the biventricular system. Equation (2) is a mixing correlation which relates the oxygen saturation of the pulmonary output stream to the oxygen saturations and flow rates of the input streams. In this equation the mixing constant, $K$, is a geometric parameter which depends primarily on the size of the ventricular septal defect. Equation (3) is an expression of the amount of oxygen extracted from the systemic circulation, based on the Fick principle (Guyton, 1971).

Like the equations for the blood flow rates, the oxygen balance equations can be expressed in threedimensional square matrices and solved for the arterial, mixed venous, and pulmonary valve infundibular oxygen saturations. Solution of the matrix equations yields the equations shows in Table 4.

\section{Computer program}

The computer program uses the equations in Tables 2 and 4 with cardiac catheterization data to determine equilibrium blood flow rates and oxygen saturation levels. The computer program is written in Fortran IV and loaded into a Fortran G compiler (Carnahan and Wilkes, 1973). The computer simulation of a patient with tetralogy of Fallot permits the variation of individual parameters to simulate the physiologic phenomena characteristic of the disease.

\section{RESULTS}

\section{Baseline model parameters}

In order to use the computer model derived for tetralogy of Fallot, it is necessary to input baseline model parameters. For this analysis, a cardiac catheterization data set for a typical child with tetralogy

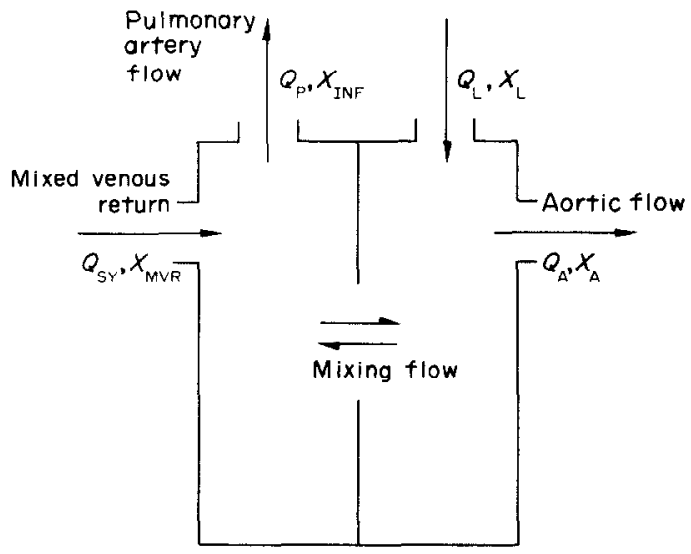

Fig. 3. Interventricular mixing model.

Table 2. Electrical circuit analog equations (see Fig. 2)

$$
\begin{aligned}
I_{1}= & \frac{E_{L H} R_{D} R_{S}+E_{R H}\left[R_{D} R_{L}+R_{D} R_{S}+R_{L} R_{P}+R_{L} R_{S}+R_{P} R_{S}\right]}{R_{D}\left[R_{P} R_{S}+R_{P} R_{L}+R_{S} R_{L}\right]+R_{S Y}\left[R_{S} R_{D}+R_{D} R_{L}+R_{S} R_{L}+R_{S} R_{P}+R_{P} R_{L}\right]} \\
I_{2}= & \frac{E_{R H}\left[R_{L} R_{P}+R_{L} R_{S}+R_{P} R_{S}\right]-E_{L H} R_{S} R_{S Y}}{R_{D}\left[R_{P} R_{S}+R_{P} R_{L}+R_{S} R_{L}\right]+R_{S Y}\left[R_{S} R_{D}+R_{D} R_{L}+R_{S} R_{L}+R_{S} R_{P}+R_{P} R_{L}\right]} \\
I_{3}= & \frac{E_{L H}\left[R_{D} R_{P}+R_{D} R_{S}+R_{D} R_{S Y}+R_{P} R_{S Y}\right]+E_{R H}\left[R_{D} R_{S}+R_{L} R_{P}+R_{L} R_{S}+R_{P} R_{S}\right]}{R_{D}\left[R_{P} R_{S}+R_{P} R_{L}+R_{S} R_{L}\right]+R_{S Y}\left[R_{S} R_{D}+R_{D} R_{L}+R_{S} R_{L}+R_{S} R_{P}+R_{P} R_{L}\right]}
\end{aligned}
$$


Table 3. Oxygen balance equations for tetralogy of Fallot

(1) $Q_{S Y}(X)_{M V R}+Q_{L}(X)_{L}=Q_{P}(X)_{I N F}+Q_{A}(X)_{A}$

(2) $K \frac{X_{L}-X_{I N F}}{X_{I N F}-X_{M V R}}=\frac{Q_{S Y}}{Q_{L}}$

(3) $\mathrm{O}_{2} \mathrm{CON}=Q_{S Y}\left[X_{A}-X_{M V R}\right] \mathrm{H}_{b} \mathrm{CAP}$

of Fallot is taken from Kirklin and Karp (1970) (see Table 5). The patient defined by this data is referred to as patient $T F$.

The input parameters to the computer program for patient $T F$ are shown in Table 6 . The oxygen saturation and oxygen consumption data come directly from the cardiac catheterization data. The mean pressures during systolic ejection are determined based on Simpson's Rule (Schwartz, 1967). The pressure drops across the right and left heart are estimated from ventricular pressure tracing data (Levin et al. 1966).

The fraction of the cardiac output which returns to the pulmonary circuit through the hypertrophied aorto-pulmonary collaterals (the aorto-pulmonary shunt fraction) is the one patient specific variable which cannot be specified from cardiac catheterization studies. For this analysis patient $T F$ is modeled at 5, 22 and 40 per cent aorto-pulmonary shunt fractions. These values represent the mean, high and low values determined by Moffit et al. (1962) and Kirklin and Karp (1970) as previously described.

Also shown in Table 6 are the baseline values for the flow rates and resistances which are calculated by the computer program. The flow rates are initially calculated based on the Fick Principle and the specified aorto-pulmonary shunt fraction. Then the individual resistances are calculated based on the basic law of blood flow:

$$
\Delta P / R=Q
$$

The value for the mixing constant, $K$, is calculated from equation (2) Table 3.

The values shown in Table 6 establish the baseline 'resting state' of the computer model for patient $T F$.

Partial factorial analysis based on the computer model

The hemodynamic model variables are divided into dependent and independent variables. The dependent variables for this analysis are the blood flow rates and the blood oxygen saturations. These are the variables which are generated by the computer simulation of the physiologic phenomena in tetralogy of Fallot.

The independent variables are the aorto-pulmonary shunt fraction, the pressure drops across the right and left heart, the circuit resistances, and the oxygen consumption. Variations in the key independent variables are specified in the computer program in order to simulate the physiology of exercise, hypoxic crisis and squatting. For this analysis the variables which are changed from the resting state in order to reproduce the above physiology are:

(1) the aorto-pulmonary shunt fraction,

(2) the oxygen consumption,

(3) the total peripheral systemic resistance, and

(4) the resistance across the stenosed pulmonary valve.

The other independent variables remain essentially constant for the computer simulations, based on the available physiologic data (Kirklin and Karp, 1970; Guyton, 1971; Wood, 1959; Guntheroth et al., 1965; Brotmacher, 1957).

The computer simulation of exercise, hypoxic crisis, and squatting in tetralogy of Fallot accurately reproduces the known effects on arterial oxygen saturation. In addition, it is possible using partial factorial analysis to demonstrate the individual effect of each key independent variable. Graphical representations of the computer output are presented which show the partial effects of the individual variables for exercise, squatting and hypoxic crisis (Figs. 4-11).

The palliative systemic-to-pulmonary shunt operation model

Palliative operations which improve the symptoms of patients with tetralogy of Fallot are employed based on constructing an artificial systemic-to-pulmonary shunt. The most common operations are the Waterston and Blalock-Taussig procedures. In order to model the revised circulatory pattern accompanying these operations, the following

Table 4. Solutions of the oxygen balance modelling equations

(1) $X_{A}=\frac{\frac{\mathrm{O}_{2} \mathrm{CON}}{\left(Q_{S Y}\right)\left(\mathrm{H}_{b} \mathrm{CAP}\right)}\left[Q_{P} Q_{S Y}-Q_{S Y}\left(Q_{S Y}+K Q_{L}\right)\right]+\left(Q_{S Y}+K Q_{L}\right)\left(Q_{L} X_{L}\right)-Q_{P}\left[K Q_{L}(X)_{L}\right]}{\left(Q_{A}-Q_{S Y}\right)\left(Q_{S Y}+K \cdot Q_{L}\right)+Q_{P} Q_{S Y}}$
(2) $X_{M V R}=\frac{\left(Q_{S Y}+K Q_{L}\right)\left[\left(Q_{L} X_{L}\right)-Q_{A}\right] \frac{\mathrm{O}_{2} \mathrm{CON}}{Q_{S Y} \mathrm{H}_{b} \mathrm{CAP}}-\left(Q_{P}\right) K\left(Q_{L} X_{L}\right)}{\left(Q_{A}-Q_{S Y}\right)\left(Q_{S Y}+K \cdot Q_{L}\right)+Q_{P} Q_{S Y}}$
(3) $X_{I N F}=\frac{\frac{\mathrm{O}_{2} \operatorname{CON}}{\mathbf{H}_{b} \mathrm{CAP} Q_{S Y}} Q_{A} Q_{S Y}+\left[K Q_{L} X_{L}\left(Q_{A}-Q_{S Y}\right)+Q_{S Y} Q_{L} X_{L}\right]}{\left(Q_{A}-Q_{S Y}\right)\left(Q_{S Y}+K \cdot Q_{L}\right)+Q_{P} Q_{S Y}}$


Table 5. Data from cardiac catheterization of a patient $T F$ with tetralogy of Fallot*

\begin{tabular}{lcc}
\hline $\begin{array}{l}\text { Catheter } \\
\text { position }\end{array}$ & $\begin{array}{c}\mathrm{O}_{2} \text { levels } \\
(\% \text { saturation })\end{array}$ & $\begin{array}{c}\text { Pressure } \\
(\mathrm{mm} \mathrm{Hg})\end{array}$ \\
\hline SVC & 54 & \\
IVC & 66 & 6 \\
RA & 61 & $102 / 0$ \\
RV sinus & 67 & $50 / 5$ \\
Infundibulum & 70 & $20 / 10$ \\
PA & & 6 \\
LA & 95 & \\
LV & 87 & $102 / 62$ \\
Aorta & 81 & \\
$\mathrm{O}_{2}$ capacity $=18.02 \mathrm{vol} \%$ & & \\
$\mathrm{O}_{2}$ consumption $=158 \mathrm{~cm}^{3} / \mathrm{min}$ & & \\
\hline
\end{tabular}

* Reproduced with permission from Kirklin and Karp (1970), p. 62.

changes are made in the baseline parameters for patient $T F$ :

(1) the aorto-pulmonary shunt fraction increases to 75 per cent;

(2) the pulmonary artery pressure increases to $30 \mathrm{~mm}$ of $\mathbf{H g}$;

(3) the arterial, mixed venous and pulmonary infundibular oxygen saturations increase to 91,71 and 84.6 per cent, respectively.

These data are based on a study of 24 patients after palliative shunting operations by Crawford et al. (1967).

\section{DISCUSSION}

Based on the computer model it is possible to simulate the following physiologic phenomena in tetralogy of Fallot:

(1) The marked decrease in arterial oxygen saturation and the over-all limitation of oxygen consumption which occur during exercise,
(2) the rapid increase in arterial oxygen saturation which occurs with squatting during recovery from exercise,

(3) the development of characteristic hypoxic spells in approximately 40 per cent of patients, and

(4) the improvement that patients experience following palliative systemic-to-pulmonary shunt operations.

\section{Exercise}

The normal physiology of the circulation during exercise is based on supplying the increased oxygen demand of the active muscle tissues. In order to supply these increased demands, both the cardiac output and the amount of oxygen extracted from each volume of blood are increased. In specific terms the normal individual requires as much as 20 times his resting oxygen consumption. In order to achieve this increase his cardiac output increases by 5-6 times and the whole body extraction of oxygen from the hemoglobin increases from 25 to 75 per cent (Guyton, 1971). Except for small amounts of blood, usually 1-2 per cent of the total cardiac output,

Table 6. Baseline parameters for Patient TF

Input parameters

$$
\begin{aligned}
& P_{S Y}=82 \mathrm{~mm} \mathrm{Hg} \\
& P_{R A}=6 \mathrm{~mm} \mathrm{Hg} \\
& P_{P A}=15 \mathrm{~mm} \mathrm{Hg} \\
& P_{L A}=6 \mathrm{~mm} \mathrm{Hg} \\
& P_{L H}=76 \mathrm{~mm} \mathrm{Hg} \\
& P_{R H}=78 \mathrm{~mm} \mathrm{Hg}
\end{aligned}
$$

Computer calculated parameters

$$
5 \% \text { Shunt }
$$

$Q_{S Y}=4 \cdot 3841 / \mathrm{min}$

$Q_{A}=4.615$

$Q_{S}=0.231$

$Q_{p}=3.378$

$Q_{D}=1.006$

$Q_{L}=3.609$

$R_{\text {SY }}=17.336 \mathrm{~mm} \mathrm{Hg} / 1 / \mathrm{min}$

$R_{S}=290 \cdot 374$

$R_{P}=20.426$

$R_{D}=1.988$

$R_{L}=2.494$

$$
\begin{array}{ll}
X_{A} & =81 \% \text { saturation } \\
X_{M V R} & =61 \% \text { saturation } \\
X_{\mathrm{INF}} & =70 \% \text { saturation } \\
& \\
\mathrm{H}_{b} \mathrm{CAP} & =18.02 \mathrm{Vol} \% \\
\mathrm{O}_{2} \mathrm{CON} & =0.158 \mathrm{l} / \mathrm{min}
\end{array}
$$

$22 \%$
$4.3841 / \mathrm{min}$
5.621
1.237
2.815
1.569
4.051
$17.336 \mathrm{~mm} \mathrm{Hg} / 1 / \mathrm{min}$
54.184
24.514
1.274
2.222

$40 \%$
$4.384 \mathrm{l} / \mathrm{min}$
7.307
2.923
1.871
2.514
4.793
$17.336 \mathrm{~mm} \mathrm{Hg} / 1 / \mathrm{min}$
22.924
36.888
1.878
0.796




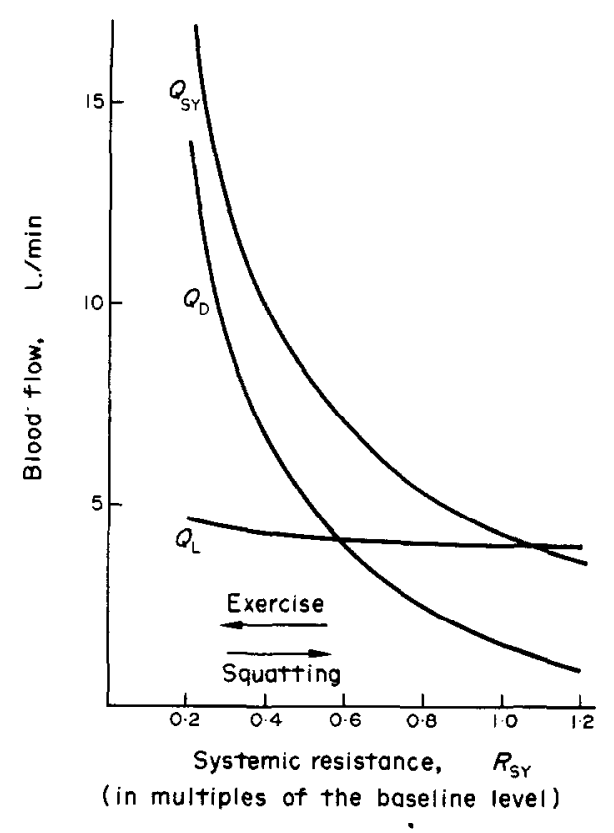

Fig. 4. The distribution of cardiac output with exercise and squatting (for patient $T F$ with 22 per cent shunt flow).

all of the increased systemic circulation passes through the pulmonary circuit. In spite of the increased flow of blood and decreased mixed venous saturation the normal individual completely oxygenates the blood leaving his lungs even during strenuous exercise. This is due to the very large pulmonary capillary surface area for diffusion, which includes reserve capacity far beyond what a normal individual requires at rest (Guyton, 1971).

In contrast the individual with tetralogy of Fallot has a tendency for arterial oxygen saturation to decrease with exercise, as well as a diminished overall ability to increase his oxygen consumption. These effects are the result of the normal physiologic responses to exercise combined with the abnormal anatomic relationships in tetralogy of Fallot. Through partial factorial analysis based on the computer model for patient $T F$ it is possible to demonstrate how these deleterious effects occur.

The key blood flow rates for patient $T F$ with a shunt flow of 22 per cent are illustrated in Fig. 4 , as predicted by the computer model. The flow rates for patient $T F$ during exercise correspond to the values for decreasing systemic resistance. This analysis is based on the fact that the most important cause of increased cardiac output with exercise is the direct vasodilation of vessels supplying metabolically active muscle tissue. Figure 4 illustrates that with increasing exercise the blood flow rates through the peripheral system and the ventricular septal defect increase markedly. In contrast the flow through the lungs remains relatively constant. Therefore, decreasing the systemic resistance causes a selective increase in blood supply to the tissues, without the corresponding normal increase in flow through the pulmonary circuit.
Oxygen consumption increases during exercise due to the increased metabolic rate of active muscle tissue. Figure $5(a)$ shows the arterial oxygen saturation for patient $T F$ at levels of exercise determined by increased oxygen consumption and decreased systemic resistance. The solid curves for constant oxygen consumption represent the physiologic range of arterial oxygen saturation and the dashed lines indicate the range where the corresponding mixed venous saturation is below equilibrium levels (Guyton, 1971). It is apparent based on Figure 5(a) that patient $T F$ is able to achieve increased oxygen consumption with decreased systemic resistance. This is because the systemic flow increases as systemic resistance decreases (Fig. 4), which allows greater oxygen transport. The most important point illustrated by Fig. 5(a) is the significant decrease in arterial oxygen saturation that occurs when the tetralogy of Fallot patient increases his oxygen consumption and decreases his systemic resistance during exercise. - The physiologic limit of increased oxygen consumption with exercise predicted by the computer simulation in approx. 3 times resting oxygen consumption, at a corresponding arterial oxygen saturation of 45 per cent (Fig. 5(a) B). This result predicted by the model correlates well with published cardiac catheterization data for patients with tetralogy of Fallot during exercise (Bing et al., 1947; Crawford et al,, 1967; Montgomery et al, 1965 and Davison et al., 1953).

Figures $5(b$ and $c)$ show the combined effects of decreased systemic resistance and increased oxygen consumption on patient $T F$ assuming 5 and 40 per cent aorto-pulmonary shunt fractions. respectively.

These graphs do not differ significantly from Fig. 5(a) for patient $T F$ with a 22 per cent shunt

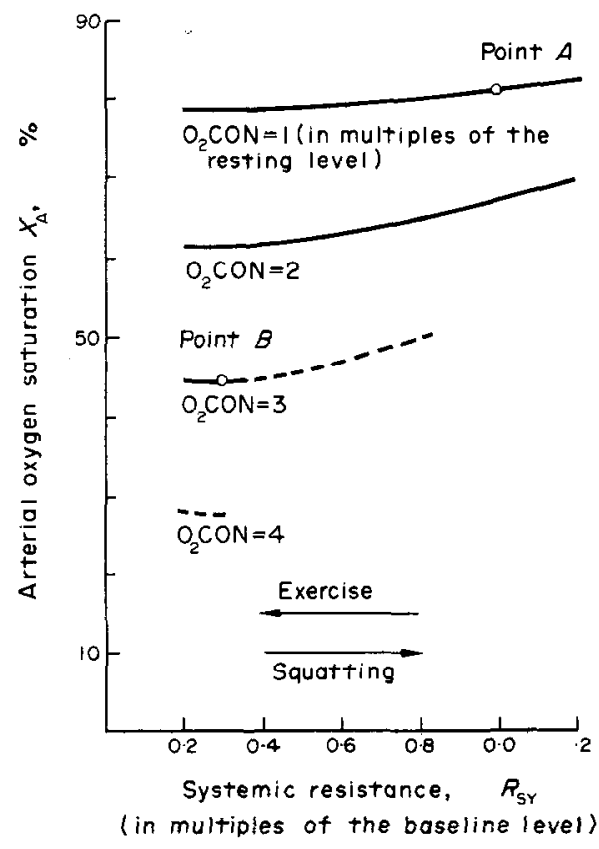

Fig. 5a. 


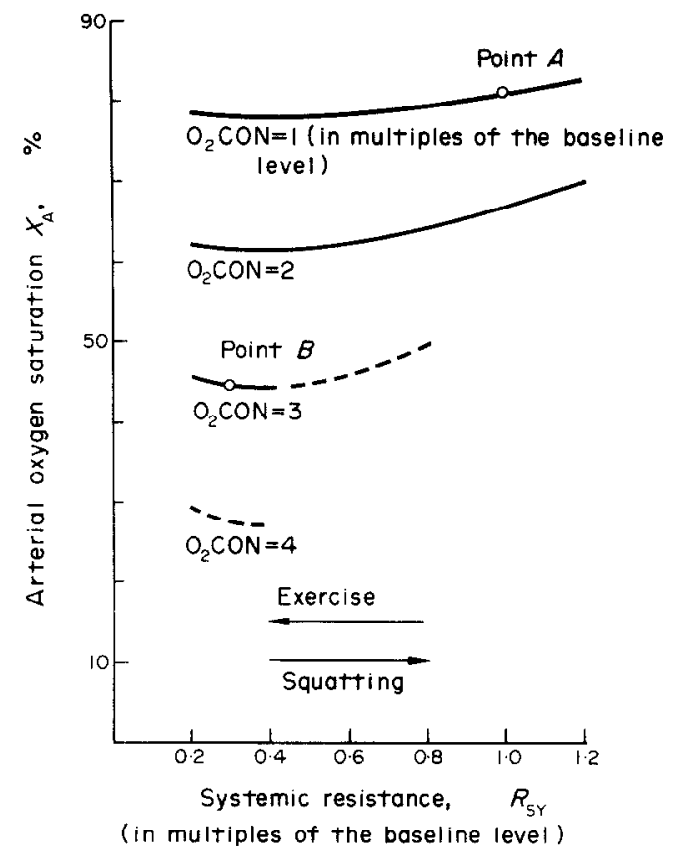

Fig. 5b.

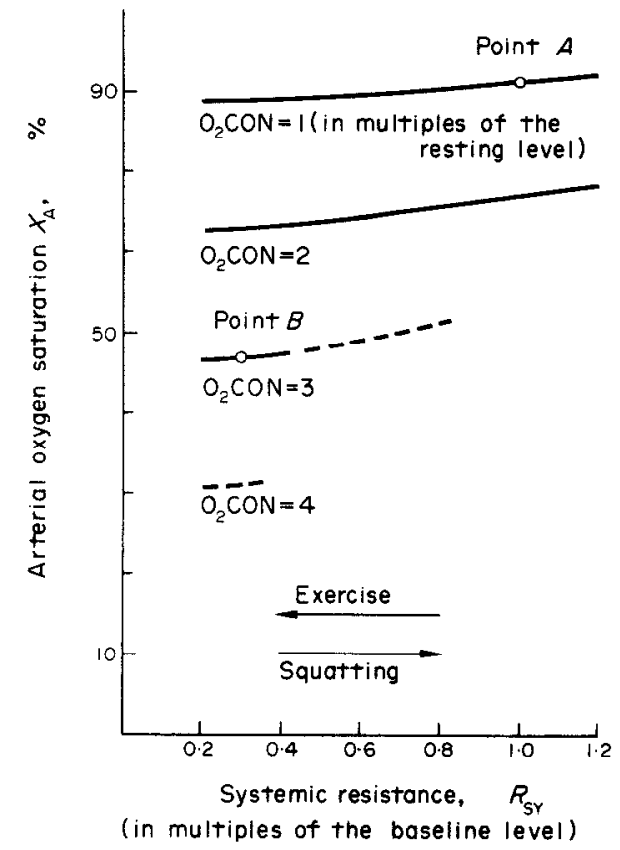

Fig. 5c.

Fig. 5. The variation of arterial oxygen saturation with exercise and squatting (a) 22 per cent shunt flow (b) 5 per cent shunt flow (c) 40 per cent shunt flow.

fraction. Therefore, it makes little difference in terms of exercise tolerance whether patient $T F$ receives most of his pulmonary flow through the pulmonary artery (Fig. 5b) or a large portion through the aortopulmonary collateral shunts (Fig. 5).

\section{Squatting after exercise}

The tendency for patients with tetralogy of Fallot to assume a squatting position following exercise is a widely recognized hallmark of the syndrome. Squatting following exercise causes a much more rapid return of arterial oxygen saturation to resting levels in the individual with tetralogy of Fallot (Brotmachev, 1957).

The mechanism by which squatting causes a rapid rise in arterial oxygen saturation is demonstrated in the computer simulation of patient $T F$. The primary hemodynamic effect of squatting following exercise has been shown by Brotmacher (1957) to be a net reduction of blood flow to the legs. This is due to a local increase in resistance caused by mechanical kinking of the femoral and popliteal vessels. The net effect of squatting after exercise is to rapidly reduce the blood flow to the active tissues of the legs back to near resting levels (Guntheroth, 1968).

Figure 4 illustrates the effect of squatting on the distribution of cardiac output for patient $T F$. Because the increased flow is primarily to the legs during lower extremity exercise, squatting will tend to directly reverse the effects of exercise. Systemic resistance will tend to increase to the resting value with a corresponding rapid fall in systemic flow relative to flow through the lungs.

During squatting after exercise the patient with tetralogy of Fallot will experience a sharp fall in oxygen consumption. This is because the highly desaturated venous return from the legs is sharply reduced by mechanical constriction of the leg vessels. The decrease in the amount of desaturated blood returning to the lungs causes a decrease in the amount of oxygen that can be transferred to the blood.

Figure 5(a) shows the effect of increasing systemic resistance and decreasing oxygen consumption on arterial oxygen saturation. It is apparent that patient $T F$ al a condition of maximal exercise represented by point $B$, will experience a sharp increase in arterial oxygen saturation with squatting. The acute reduction of blood flow to the legs due to squatting causes arterial oxygen saturation to return to near normal levels much faster than other resting positions.

An additional effect of the decrease in oxygen consumption is that restoration of the 'oxygen debt' in the leg muscles is to be deferred, in order to more rapidly increase the arterial oxygen saturation to the vital centers. As pointed out by Brotmacher (1957) this is not a problem since 'muscles that are not actively contracting can tolerate ischemia'.

Figures $5(\mathrm{~b}$ and $\mathrm{c}$ ) show that patient $T F$ will realize the same effect from squatting whether the aorta-pulmonary shunt flow is 5 or 40 per cent of total cardiac output.

\section{Hypoxic episodes}

The clinical phenomena of hypoxic episodes in patients with tetralogy of Fallot is well described by Morgan et al. (1965) based on a retrospective study of 190 cases. Thirty-eight per cent of the tetralogy paticnts had well documented histories of hypoxic episodes 'characterized by paroxysmal hypernea and increased cyanosis'. The spells were 
found to occur most often in the morning, associated with activities such as feeding, bowel movements and crying. The spells do not in general appear to occur after severe exercise. The spells were found to last 'from less than a minute to several hours, with a majority lasting 15-60 min' (Guntheroth et al., 1968). In general the patients recover from the episode, but increasing cyanosis can occur in spite of the increased respiratory effort. The patient then develops dulling of the senses, progressing to unconsciousness, convulsions and sometimes death.

Apparently the susceptibility of an individual patient to develop hypoxic spells does not correlate well with the severity of the disease, as indicated by resting arterial oxygen saturation. As Morgan et al. (1965) concluded:

'The most surprising finding was the lack of correlation of hyperpneic spells with testing arterial desaturation, although all arterial saturations obtained during attacks were quite low. Two patients with observed, typical paroxysms had arterial saturations under sedation of 93 and 98 per cent, in contrast to a patient with a 44 per cent saturation who had no spells'.

Physiologic data available from cardiac catheterization studies during hypoxic crises further defines this condition. Morgan et al. (1965) report arterial oxygen saturations of 15,24 and 33 per cent in patients tested during hypoxic spells. Wood (1959) found an average arterial oxygen saturation of 20 per cent in five cases of hypoxic crises studied during cardiac catheterization. In the same study Wood found that systemic blood pressure remains unchanged during the attacks. Hyperventilation is known to decrease systemic resistance by about 20 per cent in normal individuals (Guntheroth et al., 1965). The application of this decrease to patients

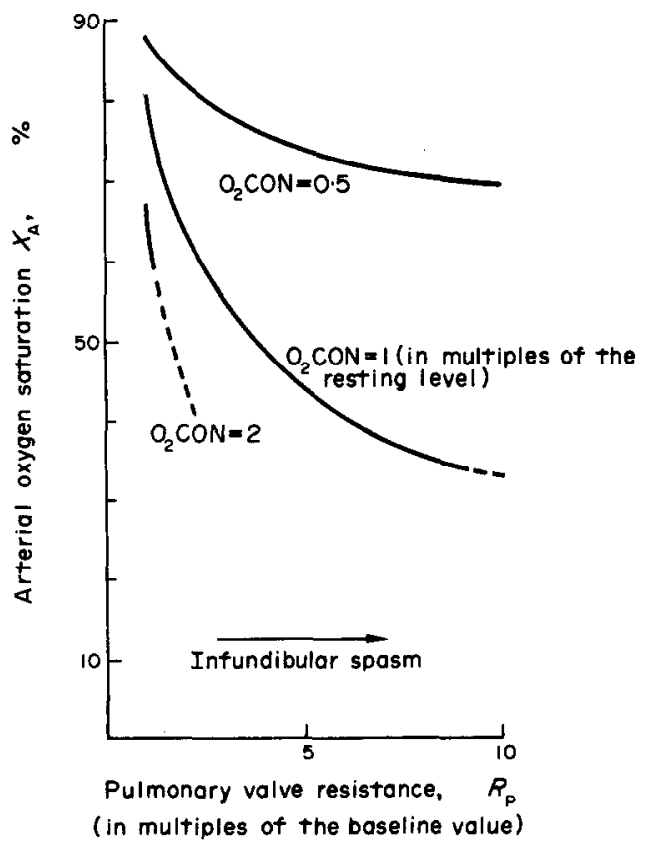

Fig. 6a.

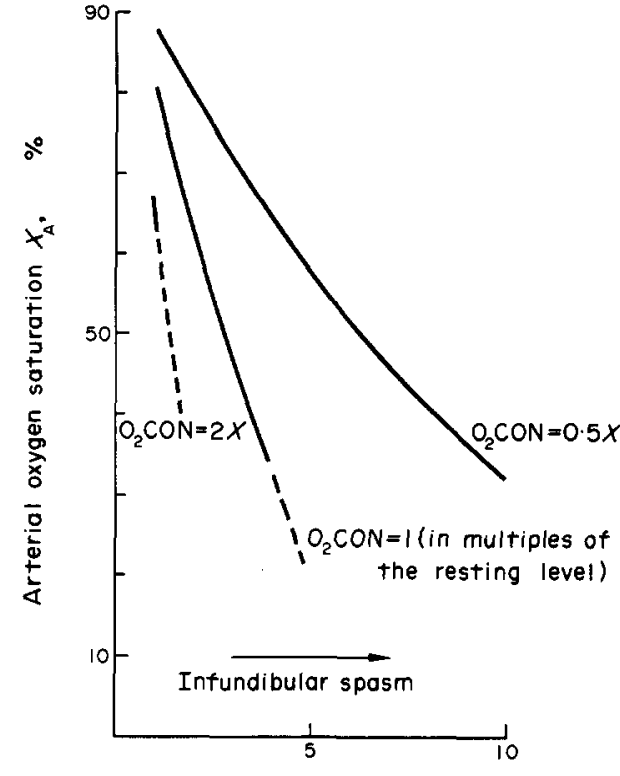

Pulmonary valve resistance, $R_{p}$ (in multiples of the baseline level)

Fig. 6 b.

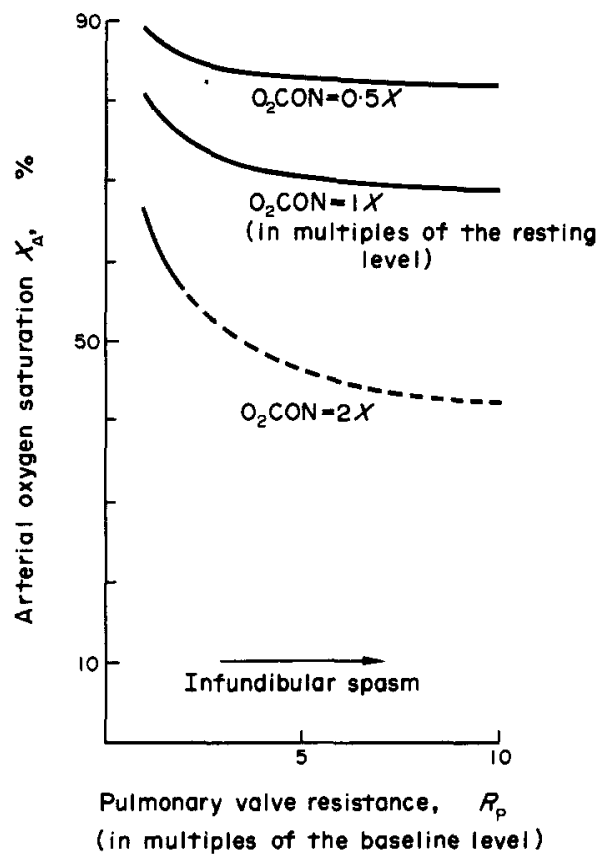

Fig. 6c.

Fig. 6. The variation of arterial oxygen saturation with increased pulmonary valve resistance (a) 22 per cent shunt flow (b) 5 per cent shunt flow (c) 40 per cent shunt now.

with tetralogy of Fallot is supported by Wood's observation that heart rate increases at constant blood pressure during hypoxic crises.

The computer model of tetralogy of Fallot provides a means to investigate the mechanism of hypoxic episodes. Wide ranges of systemic resistance, aorta-pulmonary shunt resistance, and pulmonary valve resistance have been investigated through computer simulation in an attempt to duplicate the physiologic parameters during hypoxic crises. The results 
of the analysis support the theory first advanced by Wood (1959) that hypoxic crises are caused by a sudden increase in resistance in the outflow tract of the right ventricle. An increase in resistance across the pulmonary valve is the only factor which reproduces the physiologic conditions of hypoxic crises in the computer simulation.

Figure $6(b)$ illustrates the effect of increasing the resistance across the pulmonary valve for patient $T F$ with a five per cent shunt flow. Based on hemodynamic conditions during hyperventilation described earlier, systemic blood pressure is held constant at resting values and systemic resistance is reduced 20 per cent for this analysis.

Figure $G(b)$ clearly demonstrates that the arterial oxygen saturations of approximately 20 per cent observed during hypoxic crises are caused by increased resistance of the pulmonary valve. It is also shown that increased pulmonary resistance limits oxygen consumption to less than resting capacity. This is due to the marked decrease in blood flow through the lungs (Fig. 7b). The limitation of oxygen consumption by circulatory flow rates points out the ineffectiveness of hyperventilation during hypoxic crises. In fact. mechanical work of hyperventilation has been shown to increase oxygen demand above resting levels (Morgan et al. 1965). Therefore. the response of hyperventilation to the anoxia in hypoxic crisis adversely affects the patient's overall oxygen balance. Unless the pulmonary outflow resistance is reduced naturally or with drugs, the arterial oxygen saturation decreases until vital centers are compromised.

Figures $6(a$ and $c)$ show the effect of increasing the resistance of the pulmonary valve for patient $T F$ with 22 and 40 per cent shunt flows, respectivcly. In these cases a sudden increase in pulmonary valve resistance does not induce the low arterial oxygen

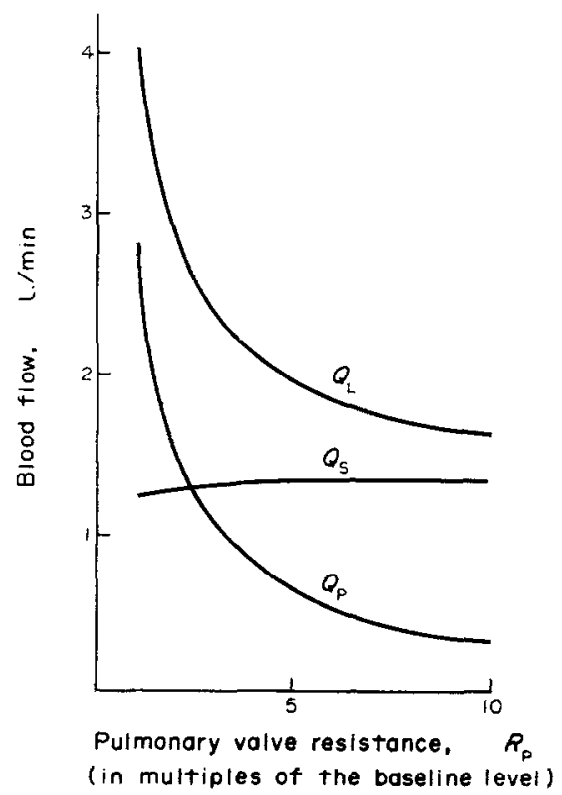

Fig. 7a.

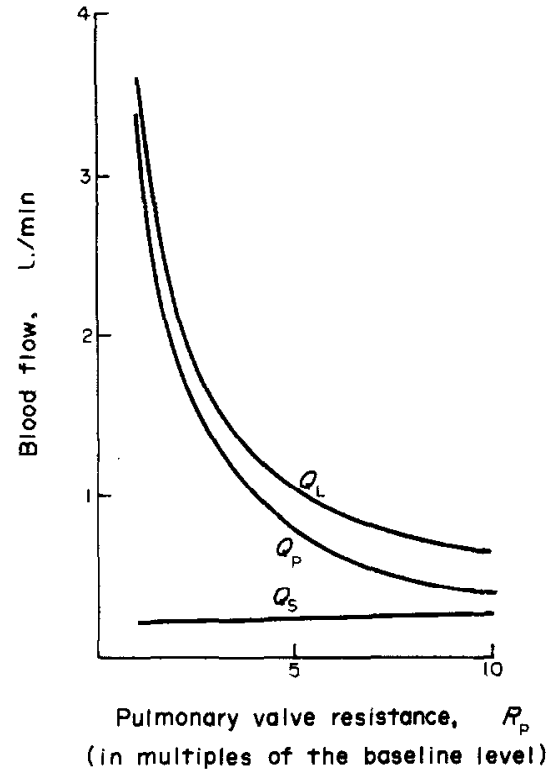

Fig. $7 b$.

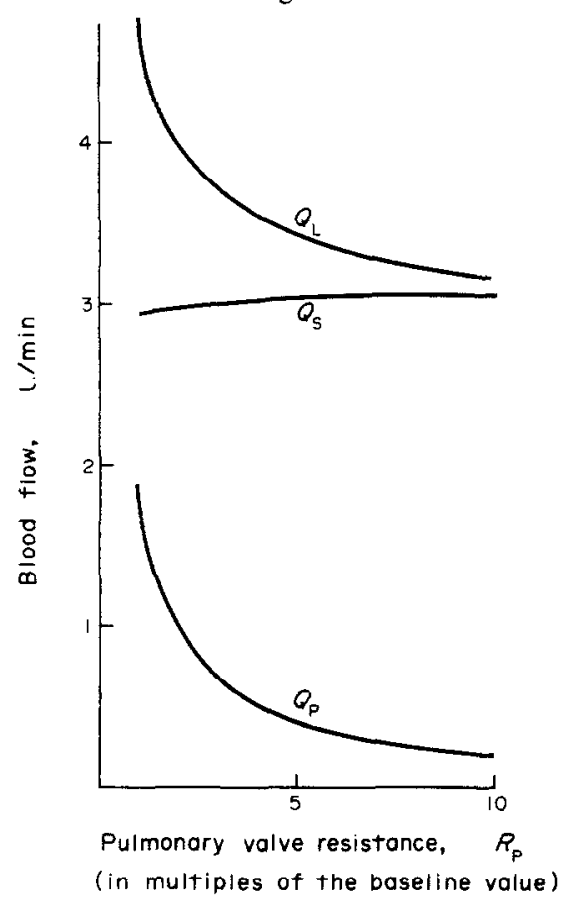

Fig. $7 \mathrm{c}$.

Fig. 7. The distribution of pulmonary blood flow with increased pulmonary valve resistance (a) 22 per cent shunt flow (b) 5 per cent shunt flow (c) 40 per cent shunt flow.

saturations found in hypoxic crises. In other words, for patients with identical resting oxygen saturations, the patient with a relatively large pulmonary infundibulum and small aorto-pulmonary shunt flow (Figure $6(b)$ ) would be susceptible to hypoxic crises while the patient with more severe infundibular restriction and larger shunt flow would not (Fig. 6(c)). The reason is shown in Figs. $7(a, b$ and c). Aorto-pulmonary shunt flow will maintain adequate flow through the lungs if the collaterals are sufficiently developed as in Figures 7(a and c). Therefore, arterial oxygen saturation will not drop to the 
low levels necessary for hypoxic crises in the patients with large aorto-pulmonary shunt flows. It is therefore demonstrated based on the computer model, why patients with tetralogy of Fallot with high resting arterial oxygen saturations may be susceptible to hypoxic crises, while patients with low resting arterial oxygen saturations may not.

\section{Palliative shunting operations}

Based on data from Crawford et al. (1967) it is possible to create a computer model of patient $T F$, after he has received a palliative shunting operation. The baseline parameters for patient $T F$ with the five per cent shunt are used, since this patient is most prone to hypoxic crises.

Figures 8 and 9 show the effects of decreased systemic resistance and increased pulmonary outflow tract resistance for patient $T F$ after a palliative shunting operation. These figures correspond to Figs. 5(b) and 6(b) for the unpalliated patient TF.

Figure 8 shows that patient $T F$ has significantly increased exercise tolerance following the operation. The graphs predict that patient $T F$ still has a significant fall in arterial oxygen saturation at maximal oxygen consumption. The magnitudes of both oxygen consumption and arterial oxygen saturation predicted in Fig. 8 from the computer simulation, correlate well with data from studies of patients after shunting procedures Crawford (1967).

Figure 9 shows that patient $T F$ is no longer susceptible to hypoxic episodes caused by increased resistance of the stenosed pulmonary valve. This graph corresponds to patient $T F$ with the worst

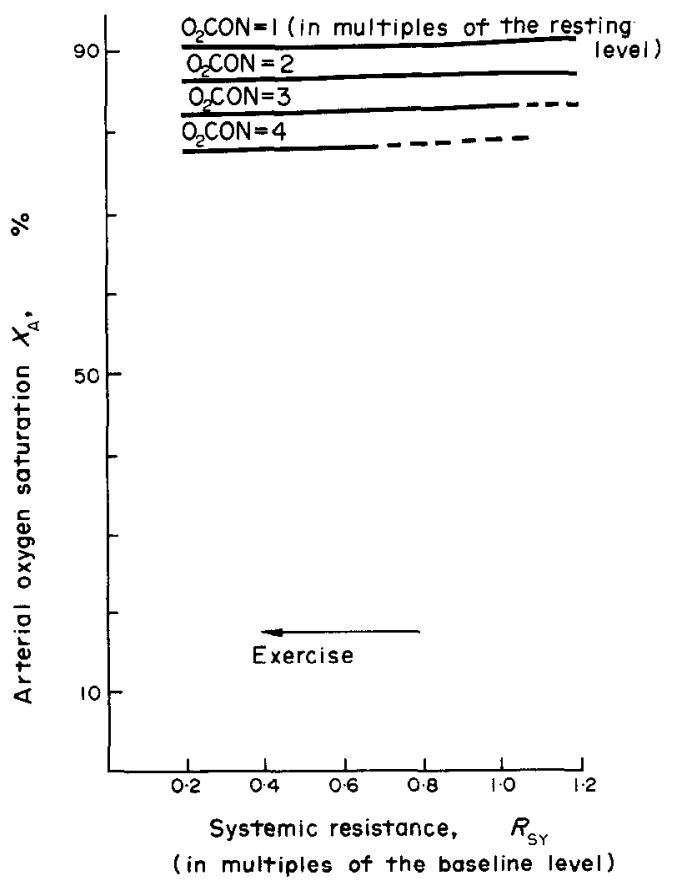

Fig. 8. The variation of arterial oxygen saturation with exercise and squatting following a palliative aorto-pulmonary shunt operation.

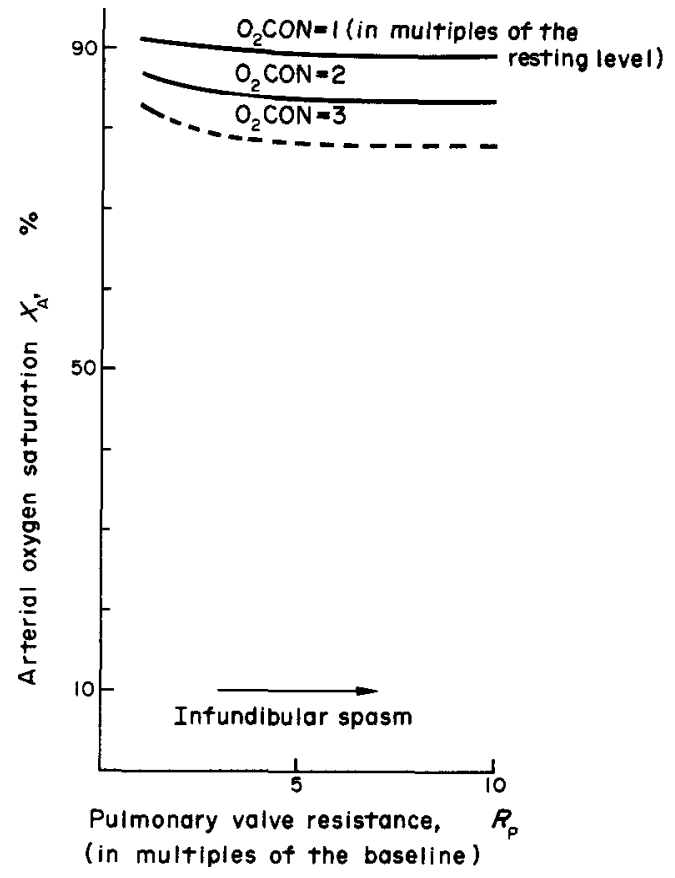

Fig. 9. The variation of arterial oxygen saturation with increased pulmonary valve resistance following a palliative aorto-pulmonary shunt operation.

prognosis in the preceding section, as shown in Fig. $5(b)$.

\section{CONCLUSIONS}

(1) The computer simulation accurately reproduces the known physiology of exercise, squatting and hypoxic episodes for an average patient with tetralogy of Fallot.

(2) The magnitude of the natural aorto-pulmonary collateral shunt flow is the most important factor in determining the susceptibility of a patient with tetralogy of Fallot to hypoxic episodes.

(3) The increase in aorto-pulmonary shunt flow following systemic-to-pulmonary operations stabilizes the arterial oxygen saturation in tetralogy of Fallot, which leads to increased exercise tolerance and decreased susceptibility to hypoxic crises.

\section{REFERENCES}

Bing, R. J., Vandam, L. D. and Bray, F. D., Jr. (1947) Physiological studies in congenital heart disease. Bull. Johns Hopkins Hospital 80, 107-141.

Brotmacher, L. (1957) Haemodynamic effects of squatting during repose and haemodynamic effects of squatting during recovery from exertion. Br. Heart. J. 19, 567.

Carnahan, B. and Wilkes, J. O. (1973) Digital Computing, Fortran IV, Watfiv, and MTS. Ann Arbor, Michigan. Crawford, D. W., Simpson, E. and Mcllroy, M. B. (1967) Cardiopulmonary function in Fallot's Tetralogy after palliative shunting operations. Am. Heart J. 74, 463.

Davison, P. H., Armitage, G. H. and Arnott, W. M. (1953) The mechanisms of adaption to a central venous-arterial shunt. Br. Heart J. 15, 21. 
Dorf, R. C. (1967) Modern Control Systems. Addison-Wesley, Reading, Massachusetts.

Guntheroth, W. G., Morgan, B. C. and Mullins, G. L. (1965) Physiologic studies of paroxysmal hyperpnea in cyanotic congenital heart disease. Circulation 31, 70. Guntheroth, W. G., Morgan, B. C., Mullins, G. L. and Baum, D. (1968) Venous return with knee-chest position and squatting in tetralogy of Fallot. Am. Heart J. 75, 313 .

Guyton, A. C. (1971) Textbook of Medical Physiology (4th edition). Saunders, Philadelphia.

Kirklin, J. W. and Karp, R. B. (1970) The Tetralogy of Fallot: From a Surgical Viewpoint. Saunders, Philadelphia.

Levin, A. R., Boineau, J. P., Spach, M. S., Coment, R. V., Jr., Capp, M. P. and Anderson, P. A. W. (1966) Ventricular pressure-Flow dynamics in tetralogy of Fallot. Circulation 34, 4.

McKerrow, C. B. and Otis, A. B. (1954) Oxygen cost of hyperventilation. Trans. Ass. Am. Phys. 67, 375.
Moffit, E. A., Kirklin, J. W. and Theye, R. A. (1962) Physiologic studies during whole-body perfusion in Tetralogy of Fallot. J. Thorac. Cardiovasc. Surg. 44, 180.

Montgomery, G. E., Jr., Geraci, J. E., Parker, R. L. and Wood, E. H. (1965) The arterial oxygen saturation in cyanotic types of congenital heart disease. Circulation $31,66$.

Morgan, B. C.. Guntheroth, W. G., Bloom, R. S. and Fyler, D. C. (1965) A clinical profile of paroxysmal hyperpnea in cyanotic congenital heart disease. Circulation 31, 66.

Schwart7., A. (1967) Calculus and Analytic Geometry. Holt. Rinehart \& Winston, New York.

Scott, R. E. (1965) Elements of Linear Circuits. AddisonWesley, Reading, Massachusetts.

Wood, P. (1959) Symposium on congenital heart disease; attacks of deeper cyanosis and loss of consciousness (Syncope) in Fallot's Tetralogy. Br. Heart J. 20, 282. 